\title{
Observed Aspects of Reconnection in Solar Eruptions
}

\author{
Ronald L. Moore • Alphonse C. Sterling • \\ G. Allen Gary · Jonathan W. Cirtain · David A. Falconer
}

Received: 30 July 2010 / Accepted: 4 February 2011 / Published online: 3 March 2011

(C) The Author(s) 2011. This article is published with open access at Springerlink.com

\begin{abstract}
The observed magnetic field configuration and signatures of reconnection in the large solar magnetic eruptions that make major flares and coronal mass ejections and in the much smaller magnetic eruptions that make X-ray jets are illustrated with cartoons and representative observed eruptions. The main reconnection signatures considered are the imaged bright emission from the heated plasma on reconnected field lines. In any of these eruptions, large or small, the magnetic field that drives the eruption and/or that drives the buildup to the eruption is initially a closed bipolar arcade. From the form and configuration of the magnetic field in and around the driving arcade and from the development of the reconnection signatures in coordination with the eruption, we infer that (1) at the onset of reconnection the reconnection current sheet is small compared to the driving arcade, and (2) the current sheet can grow to the size of the driving arcade only after reconnection starts and the unleashed erupting field dynamically forces the current sheet to grow much larger, building it up faster than the reconnection can tear it down. We conjecture that the fundamental reason the quasi-static pre-eruption field is prohibited from having a large current sheet is that the magnetic pressure is much greater than the plasma pressure in the chromosphere and low corona in eruptive solar magnetic fields.
\end{abstract}

Keywords Sun: flares · Sun: coronal mass ejections (CMEs) - Sun: magnetic reconnection - Sun: magnetic topology

\section{Introduction}

In this paper we present signatures of magnetic reconnection in solar eruptions and consider what these signatures indicate about how the reconnection develops. The solar eruptions discussed are explosive magnetic eruptions of two kinds: the ones that make major flares and major coronal mass ejections (CMEs), and the much smaller ones that make X-ray jets of the most common type, standard X-ray jets. Both kinds are magnetically driven, but

R.L. Moore (凶) · A.C. Sterling · G.A. Gary · J.W. Cirtain · D.A. Falconer NASA Marshall Space Flight Center, Huntsville, AL, USA e-mail: ron.moore@nasa.gov 
standard X-ray jets are driven in a different way from major flare/CME eruptions. Each of the two kinds of magnetic eruption has reconnection signatures that are consistent with those of the other in their implications for the reconnection. The reconnection signatures that we focus on are ribbons, loops, and strands that brighten in chromospheric and coronal movies as the eruption progresses, features made bright by plasma heating on reconnected field lines during and immediately after their reconnection.

Before there were solar telescopes in space, flares were mainly observed in the chromosphere, in $\mathrm{H} \alpha$ movies. These were studied in combination with photospheric magnetograms, maps of the polarity and strength of the magnetic flux in the flaring region. These observations showed that flares (1) happen suddenly in the magnetic field of a sunspot active region or in the remnant field of decayed active regions, (2) are centered on a neutral line (polarity inversion line) between opposite-polarity flux, and (3) have a pair of bright chromospheric ribbons that bracket the neutral line and spread apart as the flare grows (e.g., Svestka 1976; Zirin 1988). These characteristics suggested that flares are produced by sudden releases of magnetic energy. Further, together with the high electrical conductivity of the solar plasma, these characteristics gave the idea that the energy is released by a burst of reconnection at a current sheet in the corona. One of the first to have this idea was Peter Sweet (1958). Although his specific scenario for flares differs from our present understanding of solar eruptions, Sweet's basic idea that reconnection at a current sheet is the basic mechanism of magnetic energy release in flares is on very solid footing today.

The drawing on the left in Fig. 1 depicts the preflare magnetic field configuration in Sweet's model. It shows the configuration that Sweet reasoned would be formed by the magnetic fields of two identical simple bipolar active regions emerging close together, front to back. It is assumed that the emerging fields have no twist so that for either active region emerging alone in the absence of any pre-existing field, the emerged field would relax to
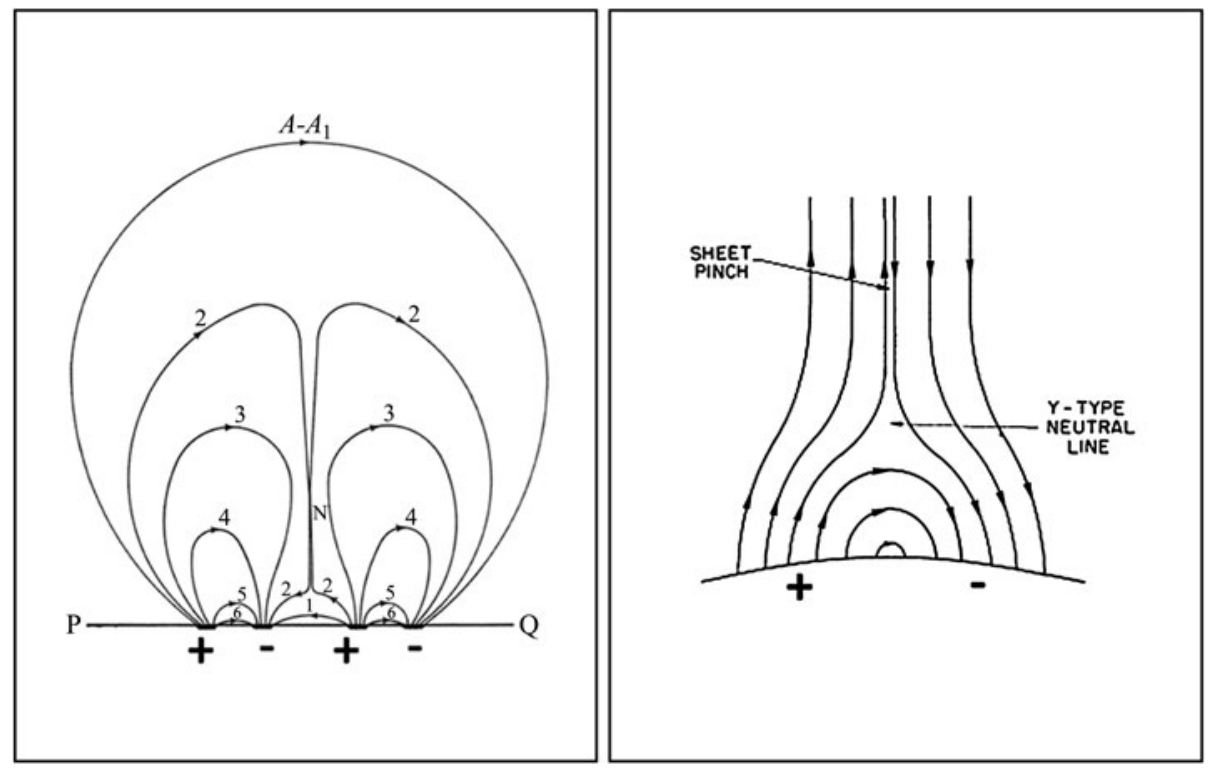

Fig. 1 Schematic depictions of the preflare magnetic field in early flare models. Left: Quadrupolar model of Sweet (1958). Right: Bipolar model of Carmichael (1964) and Sturrock (1966, 1968). These drawings are from Sweet (1958) and Sturrock (1968). We have added the plus and minus signs marking the flux polarities 
its minimum-energy potential-field configuration, the configuration that is devoid of electric current and that therefore has no free magnetic energy. But when the two active regions emerge close together, the overall quadrupolar field is prevented from relaxing to its potential-field configuration because the field and the plasma in each arch are frozen together by the plasma's high electrical conductivity. In Sweet's model, the two emerging arches are lined up and have the positive-polarity end of one next to the negative-polarity end of the other. In pushing against each other the two arches build up a current sheet at their interface between the two impacted legs of oppositely-directed field. Figure 1 (left) portrays the two compressed fields sandwiching the current-sheet interface. In this quadrupolar preflare field configuration, the field is all closed.

The work done in building the current sheet becomes the free energy of the compressed fields. A stressed field of this configuration and of the strength and size of the magnetic field in solar active regions could have enough free energy for a major flare. In this scenario, the current sheet builds up until reconnection sets it. The ensuing burst of reconnection relaxes the configuration closer to the potential-field configuration by releasing free magnetic energy to become the energy of plasma flows and nonthermal particles, much of which dissipates into heat on the reconnected field lines. Because the reconnecting field lines are closed, the reconnection heating makes four flare ribbons, a pair that is at the feet of the reconnected field lines on the outside of the quadrupole and that brackets all three neural lines, and an inside pair that brackets only the middle neutral line, the one between the two emerging magnetic arches.

Some flares do have four ribbons, but it was observed that major flares typically have only two, bracketing a single neutral line (e.g., Bruzek 1964). So, the next idea was to have only a single bipolar active region emerge, and then have much of the magnetic arch gradually pulled open by the solar wind (Carmichael 1964; Sturrock 1966, 1968). This would build an inverted-Y open field configuration having a vertical current sheet at the interface between the two opposite-polarity opened legs of the magnetic arch, as in the sketch on the right in Fig. 1. The buildup is supposed to continue until there is enough free energy in the opened field for a major flare. Then reconnection sets in at the current sheet. The reconnection of the open field makes both (1) closed field lines that are released downward, building a growing flare arcade that heats a flare ribbon at each foot, and (2) open-field U loops that are released upward, propelling a huge plasmoid into the outer corona and solar wind. In the model proposed by Sturrock $(1966,1968)$, the ejected plasmoid was roughly what would later be known as a CME (Rust et al. 1980).

The thrust of this paper is that the early flare models sketched in Fig. 1 are wrong in one big way for major flare/CME eruptions. These eruptions are indeed explosive releases of magnetic energy, and these magnetic explosions are indeed unleashed by reconnection at one or more current sheets. But when the reconnection starts, there is no evidence of a large current sheet like those in Fig. 1, no current sheet of extent comparable to that of the overall pre-eruption field configuration. Instead, at the start of the reconnection early in the eruption, the observed spatial extent of reconnection heating is small, indicating that the reconnection current sheet is small compared to the overall size of the erupting field. Rapid growth of the spatial extent of the reconnection heating as the eruption explodes indicates that reconnection progressively further unleashes and speeds up the eruption, and that the growing eruption in turn forces the reconnection current sheet to grow much larger and reconnect flux at a much greater rate. These observations imply that most of the free magnetic energy released in a major flare/CME eruption is not built up by the gradual building of a large current sheet in the pre-eruption field as in Fig. 1. Instead, it appears that most of the free energy to be released is in sheared and twisted field in the core of a bipolar arcade, and 
that there is no large-scale current sheet until the eruption is well underway and has become highly dynamic.

In the following sections, we support the above points by presenting representative examples of observed solar eruptions and their reconnection signatures. Most of the space is devoted to four examples of major flare/CME eruptions. In two of these, the field that erupts is an isolated sheared-core arcade. That is, the erupting active region is a bipolar active region that is far from other active regions. In the other two, the field that erupts is a sheared-core arcade embedded in the middle of a quadrupolar active region. In addition to the example flare/CME eruptions, we present an example of another kind of solar eruption, a standard X-ray jet. In the pre-eruption field for these eruptions, the free energy to be released is not in the sheared core of a bipolar arcade. Instead, it is in the compressed field around a current sheet built by the gradual pushing together of two disparate fields as in Sweet's model (Fig. 1, left), but with the impacting fields being an emerging magnetic arcade and extant high-reaching unipolar field. In agreement with the implications of the reconnection signatures of major flare/CME eruptions, the reconnection signatures of standard X-ray jets imply that the growing pre-eruption current sheet is still small on the scale of the overall field configuration when reconnection sets in and unleashes the eruption. For each of the three varieties of eruption (sheared-core isolated arcade, sheared-core embedded arcade, and standard jet), before showing the observations we present cartoons depicting the field configuration, the reconnection, and the resulting signatures seen in the observations.

\section{Reconnection in Flare/CME Eruptions}

\subsection{Isolated-Arcade Eruptions}

The drawings in Fig. 2 depict in 3D the now standard scenario for the eruptions that produce major flares and major CMEs. In any solar eruption that produces a major flare, whether or not the eruption produces a CME and whether or not the erupting field is embedded in comparably strong surrounding field, the field that erupts is a bipolar arcade that is basically of the form sketched in the upper left panel of Fig. 2. The core of the pre-eruption arcade, the field rooted near the arcade's neutral line, is strongly sheared and twisted so that it roughly traces the neutral line and typically has an overall sigmoid form like that in the drawing. The shear in the arcade's field decreases with distance from the neutral line, so that the outer envelope has little shear. As in this cartoon, the pre-eruption core field often holds a filament of chromospheric-temperature plasma. The filament is suspended by nearly horizontal field lines (not shown) that thread the filament and are strands of what basically amounts to a flux rope (not shown) that runs the length of the sigmoid. The core field is directed nearly parallel to the neutral line whereas in the potential-field configuration it would be nearly orthogonal to the neutral line. This signifies that the core field is so greatly deformed from the potential field that most of its magnetic energy is free energy (e.g., Svestka 1976; Heyvaerts et al. 1977; Sturrock 1980; Zirin 1988; Moore 1988; Moore and Sterling 2006). This pent-up free energy renders the core field or even the entire arcade capable of erupting.

Major flares typically occur in strong-field regions, regions in which the magnetic pressure is much greater than the plasma pressure in the chromosphere and low corona, and the weight and pressure of the plasma are negligible in the overall force balance of the pre-eruption field. Figure 2 shows the case of the pre-eruption arcade standing alone in the absence of any appreciable other overarching field rooted around it. In this case, the core field is partly restrained from expanding upward by the downward pull of its own field lines 


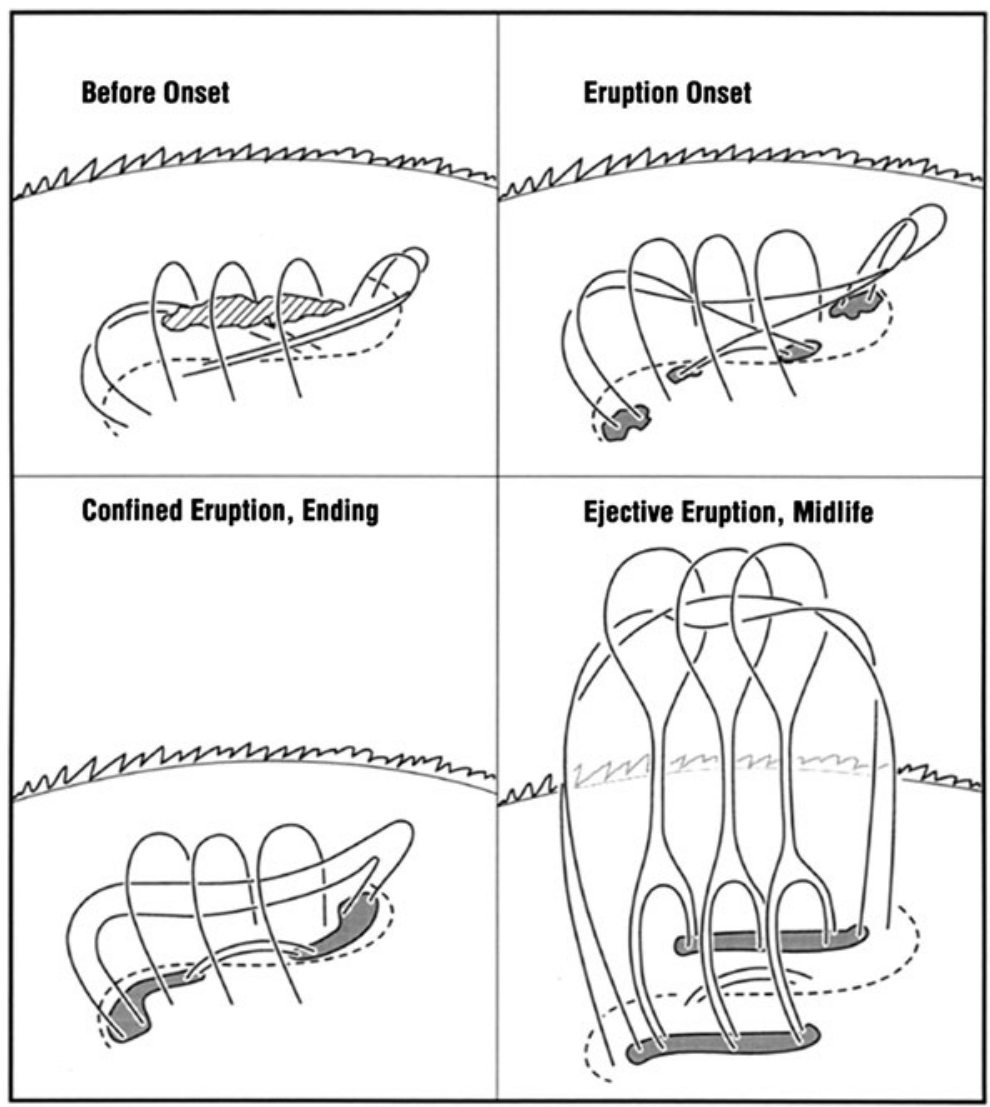

Fig. 2 Progression of the three-dimensional configuration of the driving magnetic field and its internal reconnection in major flare/CME eruptions (from Moore et al. 2001). Only a few representative field lines (solid curves) are drawn, showing only the rudiments of the field. The dashed curve is the magnetic polarity inversion line. The cross-hatched elongated feature in the first panel is a filament of cool plasma suspended in the sheared core field. The filament plasma is carried in the erupting core-field flux rope, but is not shown in the other panels for clarity of the field configuration. The shaded areas are flare ribbons at the feet of reconnection-heated field lines. The ragged arc in the background is the chromospheric limb of the Sun

anchored in the photosphere, but it is mostly restrained by the arcade's envelope holding it down. When the arcade is embedded in appreciable overarching field, the downward force of that field helps the arcade hold down the core field. In general, before eruption onset, the core field, the rest of the arcade field, and any overarching field are in a metastable equilibrium in which the overall upward push of magnetic pressure is balanced by the overall downward pull of magnetic tension. The eruption begins when this equilibrium is lost or broken, so that the tension no longer completely restrains the pressure, allowing the core field to begin erupting upward (Moore and LaBonte 1980; Moore and Roumeliotis 1992; Isenberg et al. 1993; Antiochos 1998; Chen and Shibata 2000; Klimchuck 2001; Moore and Sterling 2006). The particulars of the field configuration and its evolution determine the mechanism or combination of mechanisms that breaks the equilibrium and triggers the eruption. As is laid out in Moore and Sterling (2006), if the eruptive arcade is embedded 
in opposite overarching field (not shown in Fig. 2), then there is the following range of possibilities for the trigger:

1. Tether-cutting reconnection: reconnection between the sheared legs of the sigmoid (as depicted in the upper right panel of Fig. 2) or between extant field and emerging field anywhere inside the arcade (Moore and Roumeliotis 1992).

2. Breakout reconnection: reconnection between the arcade and overarching opposite field (Antiochos 1998).

3. MHD instability: any magnetically driven eruptive process that begins without reconnection (Forbes 2000). For example, kink instability of the core-field flux rope (Rust and Kumar 1996).

4. Any two of the above three mechanisms, or even all three, acting together.

If the eruptive arcade is isolated from other field so that it stands alone as depicted in Fig. 2, breakout reconnection is not an option. The possibilities for the trigger are then (1) tether-cutting reconnection, (2) MHD instability, or (3) these two mechanisms acting in concert.

In an isolated-arcade eruption, tether-cutting reconnection below the erupting core-field flux rope starts together with the eruption or soon after and progressively further unleashes the eruption. This reconnection (1) adds heated strands of field to the erupting flux rope above it, and (2) makes hot flare loops below it. At first, the reconnection current sheet and the heated strands, loops, and ribbons are small, as in the upper right panel of Fig. 2, but they grow in step with the eruption. If the envelope of the arcade is strong enough, the eruption is confined. The arcade stays closed and the eruptive motion and tether-cutting reconnection soon stop as in the lower left panel of Fig. 2. If the growing erupting flux rope is strong enough, the eruption is ejective. The eruption and reconnection continue to grow explosively. The erupting flux rope and arcade envelope blow out to become a CME as the current sheet, flare arcade, and pair of separating flare ribbons grow to become comparable in span to the pre-eruption arcade, as indicated in the lower right panel of Fig. 2. As Fig. 2 indicates, in isolated-arcade eruptions, regardless of whether the eruption is confined or ejective, the preeruption field configuration and the small extent of the flare loops and ribbons early in the eruption show that no current sheet of the size of the arcade is present at the start.

Figure 3 shows the filament eruption and the onset and growth of the chromospheric $\mathrm{H} \alpha$ flare ribbons observed in a famous isolated-arcade ejective eruption. This large, longduration, two-ribbon flare occurred in a decayed bipolar active region that no longer had sunspots and that was about $45^{\circ}$ east of central meridian. In addition to being well observed in ground-based magnetograms and $\mathrm{H} \alpha$ movies, this active region and flare were observed from space by Skylab in coronal X-ray images taken before and after the eruption. These observations showed that there were no other active regions nearby, and that the pre-eruption magnetic field was a bipolar arcade like that sketched in Fig. 2. The core field was obviously strongly sheared, was sigmoidal in overall form, and had a large filament suspended in it (Moore and LaBonte 1980).

In the first four frames of Fig. 3, the filament is on the verge of erupting. As can be seen from inspection of the height of the bottom of the filament above the dark fibril on the neutral line below it, over the 37 minutes spanned by the first four frames of Fig. 3, the filament gradually rose to about twice its initial height. This pre-eruption slow rise ended during the 7-minute interval between the fourth and fifth frames. In the fifth frame, the filament is about twice higher than in the previous frame, and is rapidly rising and disappearing as the pair of flare ribbons become faintly discernible below it. Here at their onset, the two ribbons are faint, are much smaller in extent than the filament and arcade, are close against the neutral 

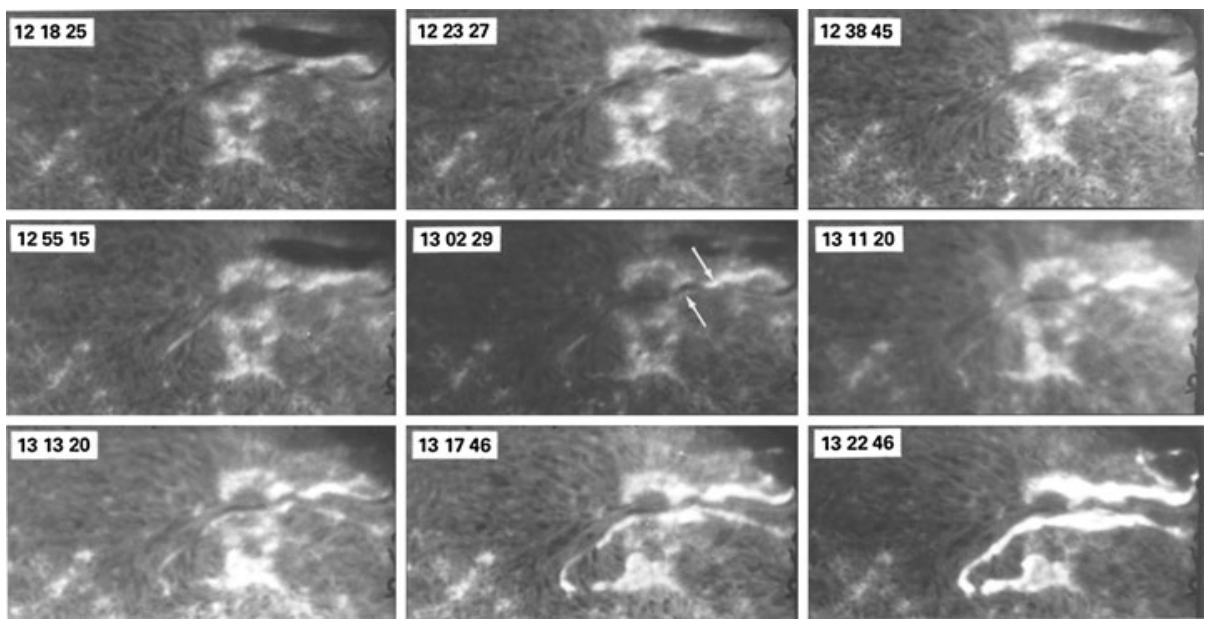

Fig. 3 Filament lift-off and onset and growth of the flare ribbons in the explosive phase of the large two-ribbon flare of 1973 July 29. This sequence of H $\alpha$ images is presented in Moore and LaBonte (1980). It covers the pre-eruption slow rise of the filament and the growth of the flare ribbons from their start as the filament erupts. Each image is labeled with its universal time. The filament is about $80,000 \mathrm{~km} \mathrm{long}$. In the middle frame, the arrows point out the beginnings of the conjugate pair of flare ribbons along the neutral line under the erupting filament

line, and are offset from each other along the neutral line, which is traced by the dark fibril between them. Over the next 20 minutes in Fig. 3 (fifth frame to last frame), the two ribbons grow increasingly faster in brightness, width, and length, growing to span the length of the arcade as they spread away from the neutral line. After their explosive growth seen in Fig. 3, the two flare ribbons more gradually spread away from the neutral line, decelerating over the next 12 hours, becoming shorter, thinner, and fainter as the X-ray flare arcade rooted in them gradually grew taller and fainter (Moore et al. 1980). That the flare lasted this long certifies that the eruption was ejective and became a CME (Sheeley et al. 1983). Thus, the $\mathrm{H} \alpha$ images in Fig. 3 show good evidence that the ribbon-heating reconnection current sheet formed and grew as in Fig. 2, forming under the filament as the core-field flux rope started erupting, initially being small, and then growing much larger as the eruption blew open the arcade.

Our second example isolated-arcade eruption produced a major X-ray flare (GOES M3) and was well observed in coronal X-ray images from the Yohkoh Soft X-ray Telescope (SXT). The upper four panels of Fig. 4 are snapshots from SXT that show the active-region arcade before and during the eruption and the flare arcade hours after the eruption. Because the active region was on the central disk, at north $5^{\circ}$ and west $25^{\circ}$, these images view the arcade mostly from above, but somewhat from its east (left) side. The bottom left panel of Fig. 4 is a magnetogram of the area viewed in the X-ray images. It shows that this sunspot active region was largely bipolar and had no other active regions near it, verifying that the eruptive magnetic field was an isolated bipolar arcade.

The first X-ray image in Fig. 4 shows the arcade a few hours before the eruption. Together with the magnetogram, it shows that the core field was sheared and sigmoidal in the manner of Fig. 2. The envelope of the arcade is faint and indistinct in this image. Arrows A and C point to the two elbows of the sigmoid core field, and arrows B1 and B2 point to faint loops of the envelope. The second X-ray image in Fig. 4 catches the eruption during its onset, at about the stage depicted in the second panel of Fig. 2. It shows the expected signatures 

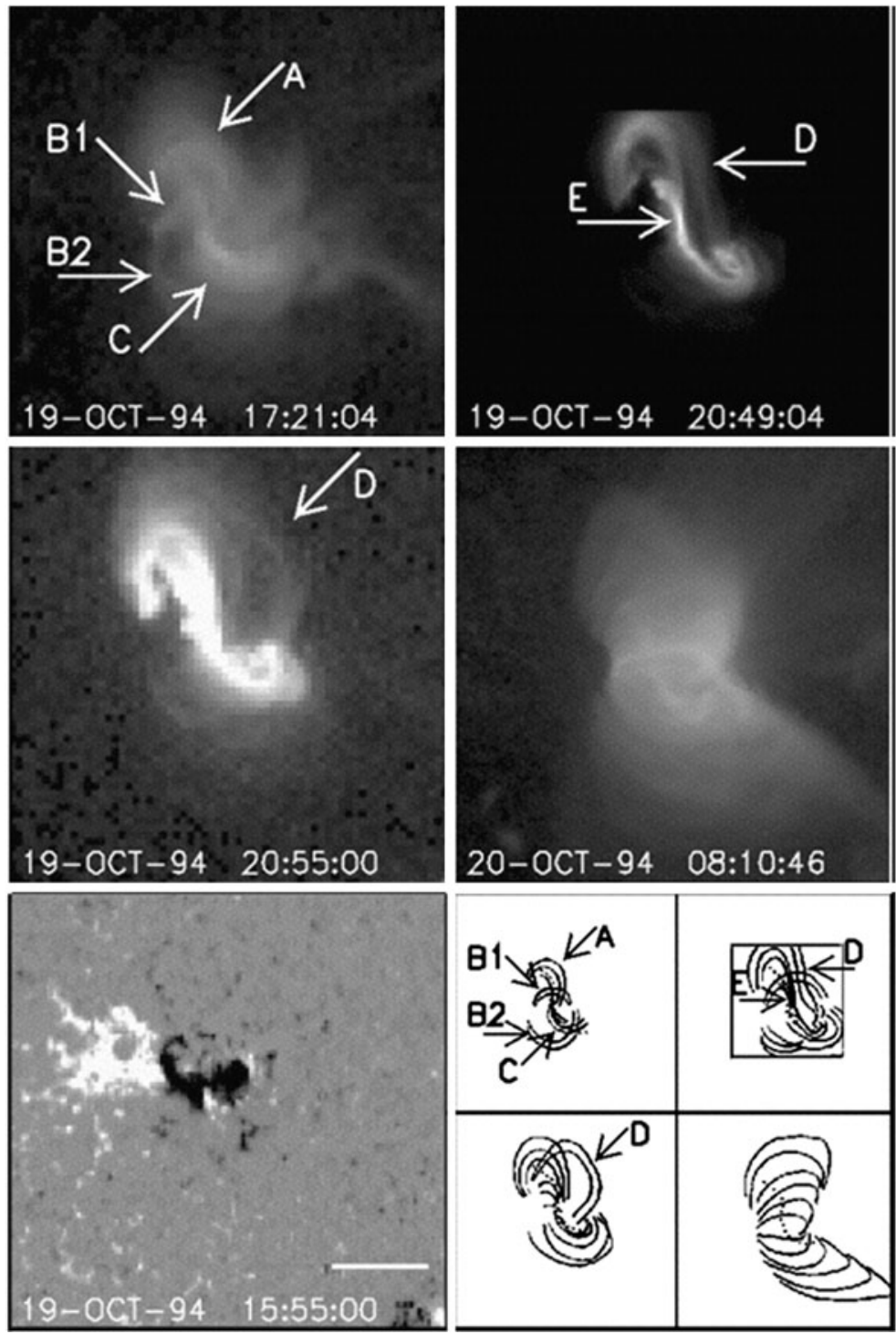

Fig. 4 Onset and growth of the X-ray flare arcade in step with the eruption of the core-field flux rope in an isolated-arcade ejective eruption on 1994 October 19 (from Moore et al. 2001). The upper four panels are X-ray images from Yohkoh/SXT. The bottom left panel is a co-spatial photospheric magnetogram from NSO/Kitt Peak, mapping the positive (light) and negative (dark) magnetic flux in and around the active region. The horizontal white bar in the magnetogram is $100,000 \mathrm{~km}$ long. The date and universal time are given in each image. The bottom right panel is a "road map" showing the neutral line (dotted curve) traced from the magnetogram and selected loops (solid curves) traced from the X-ray images. North is up and west is right in these and all subsequent solar images in this paper

of reconnection occurring at a small current sheet in the sheared field low in the middle of the sigmoid: (1) the small sheared bright loops of the start of the flare arcade below the reconnection site (pointed to by arrow E), and (2) heated bright field strands that run 
the length of the sigmoid and are escaping upward in an erupting flux rope (pointed to by arrow D). Six minutes later, in the third X-ray image in Fig. 4, the flux rope has erupted farther up while the still strongly sheared flare arcade has grown much brighter and much more extensive, consistent with explosive growth of the reconnection current sheet in step with the eruption. The last X-ray image in Fig. 4 shows the full-grown fading X-ray flare arcade late in the flare's decay phase (Moore et al. 2001), about 11 hours after the eruption blew open the arcade and spit out a CME plasmoid that escaped into the solar wind. At this late stage, the flare arcade has very little shear, in agreement with the flare arcade being formed by reconnection reclosing of the blown-out envelope of the arcade as depicted in the fourth panel of Fig. 2. In this isolated-arcade eruption, the pre-eruption arcade was similar in form and spatial extent to that of the previous example isolated eruptive arcade, but the field was stronger because the active region in the present example still had sunspots. Therefore, it is reasonable that the present eruption and its reconnection current sheet probably grew faster than the previous eruption and its reconnection current sheet, as comparison of the growth of the flare ribbons in Fig. 3 with the growth of the flare arcade in Fig. 4 suggests. The sigmoid-to-arcade progression of the X-ray flare structure in Fig. 4 and the long duration of the flare certify that the present eruption was ejective and blew out a CME in the manner depicted in Fig. 2 (Sheeley et al. 1983; Canfield et al. 1999; Sterling et al. 2000). Thus, the X-ray images in Fig. 4 are nicely consistent with the $\mathrm{H} \alpha$ images in Fig. 3, and show evidence at least as strong that the reconnection current sheet was formed as indicated in Fig. 2, starting small and growing much larger in step with the eruption.

\subsection{Embedded-Arcade Eruptions}

Often, the sheared-core arcade that erupts is not the field of an isolated bipolar active region, but is embedded in the field of a larger active region having one or more other major neutral lines. Sometimes the whole multipolar active region is quadrupolar, having three neutral lines, one of which is the neutral line of the eruptive arcade. The cartoon on the left in Fig. 5 is a $3 \mathrm{D}$ topological sketch of the field of an ideal symmetrical quadrupolar active region in which the four flux domains of alternating polarity are equal in flux and equal in area, and are packed against each other along a straight line. For this flux arrangement, the field has three embedded bipolar arcades, one for each neutral line. Any of these arcades can erupt if its core field has enough free energy, enough shear and twist. Sometimes it is one of the two side arcades that erupts, sometimes it is the central arcade, and sometimes first one arcade erupts and then another. The cartoons in Fig. 5 are for the cases in which only the central arcade erupts or the central arcade erupts first. These cartoons symbolically depict the central arcade's sigmoidal sheared and twisted core field that is needed for eruption and ignore any such nonpotential field that may be present in the side arcades. In the 3D cartoon in Fig. 5, the pre-eruption sigmoidal core field in the central embedded arcade is like the core field in the isolated arcade in Fig. 2 except that here the core field's shear and twist is right-handed whereas in Fig. 2 it is left-handed. The $2 \mathrm{D}$ cartoons on the right in Fig. 5 depict this 3D quadrupolar setup viewed end-on.

In a quadrupolar active region such as sketched in Fig. 5, the field in the overarching outer envelope is opposite in direction to the field in the envelope of the underlying eruptive central arcade. In this situation, in addition to the possibilities of internal tether-cutting reconnection and MHD instability for triggering the eruption of the middle arcade, there is also the possibility of triggering the eruption by breakout reconnection at the top of the central arcade. The three 2D cartoons in Fig. 5 depict the onset and explosive progression of the eruption when the eruption starts by breakout reconnection. The evolutionary buildup 

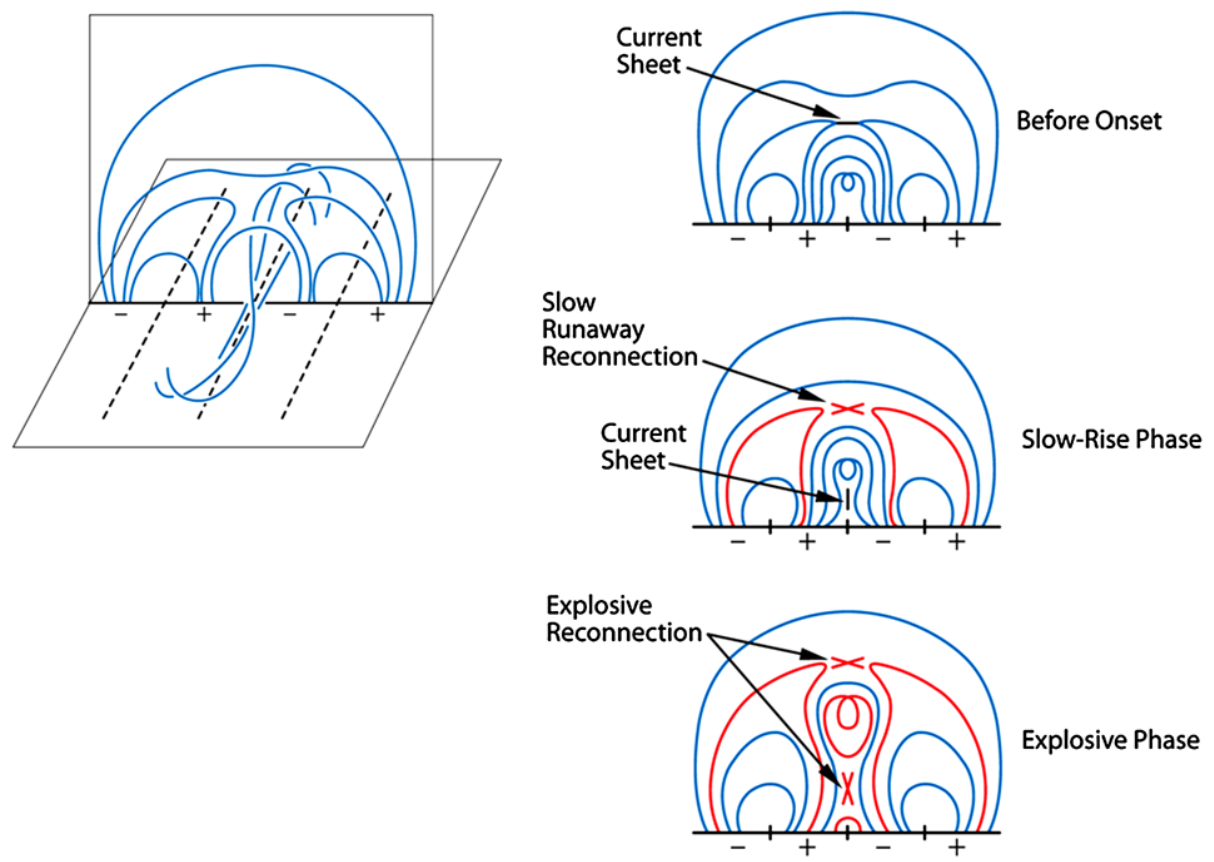

Fig. 5 Magnetic field topology and progression of reconnection in embedded-arcade ejective eruptions in which the eruptive arcade is the central arcade of a quadrupolar active region and the eruption is started by breakout reconnection (from Moore and Sterling 2006). Left: 3D cartoon of the field of a symmetric quadrupolar active region in which the central embedded arcade has enough free energy in its sigmoidal core field to undergo a CME-producing ejective eruption. As in Fig. 1, only a few representative field lines (blue) are drawn. Each dashed line is a polarity inversion line in the photospheric magnetic flux. Right: 2D cartoons showing the unleashing of the eruption by breakout reconnection and by tether-cutting reconnection. Each of the two current sheets is shown by a short black line when it has formed but has not yet become unstable to reconnection. Reconnection in progress at a current sheet is marked by a red X. Red curves are field lines on which the plasma is hot $\left(T \gtrsim 3 \times 10^{6} \mathrm{~K}\right)$ from heating by reconnection. Blue curves are field lines that have not yet or will not undergo reconnection

of free energy in its sigmoid core causes the central arcade to gradually puff up and push against the overarching opposite field, building a current sheet at the interface as depicted in the top 2D cartoon (e.g., Lynch et al. 2008). When this current sheet grows to be wide enough and thin enough, it becomes unstable to reconnection and breakout reconnection begins there. The reconnected field springs aside, the downward force that held the central arcade in check is reduced, and the central arcade begins erupting upward. The central arcade then presses harder on the overarching field, forcing the current sheet to grow larger and reconnect faster, further unleashing the central arcade to erupt upward (Antiochos et al. 1999; Lynch et al. 2008). The middle 2D cartoon depicts this growing runaway breakout reconnection and upward eruption soon after onset. The field lines that are reconnected and heated by the breakout reconnection are new hot loops on the outside of each side arcade. This makes four flare ribbons, one at each foot of these two hot arcades. These two side-arcade flare loops and ribbons are the key signature of the breakout reconnection. These features are localized at first and grow in lateral extent as the eruption speeds up, indicating that the breakout reconnection starts when the current sheet is still small on the scale of the erupting arcade. 
The middle 2D cartoon in Fig. 5 also depicts that as soon as the arcade erupts far enough, its two stretched legs become pressed together and make a current sheet between them. Tether-cutting reconnection soon starts and grows at this current sheet as in isolated-arcade eruptions (as in Fig. 2). This reconnection, along with the breakout reconnection, further unleashes and speeds up the eruption, which, in turn, forces both current sheets to grow further and reconnect faster (Lynch et al. 2008). This most explosive phase of the eruption is depicted in the bottom 2D cartoon in Fig. 5. The tether-cutting reconnection of the legs of the erupting central arcade makes a growing flare arcade and pair of separating flare ribbons centered on the neutral line of the erupting arcade as in an isolated-arcade eruption. Sometimes there is this quadrupolar setup that allows breakout reconnection, but when the central arcade erupts to produce a CME the breakout reconnection is too weak to heat the side-arcade flare loops and ribbons enough for them to be seen. But in any CME-producing eruption of an embedded arcade in an active region, an obvious growing flare arcade and its flare ribbons develop along the neutral line as in an isolated-arcade eruption, starting small early in the eruption and growing much larger as the arcade blows out.

Our first example embedded-arcade eruption is shown in Fig. 6. The top four panels are $\mathrm{SOHO/EIT} \mathrm{Fe} \mathrm{XII} \mathrm{coronal} \mathrm{images} \mathrm{of} \mathrm{the} \mathrm{active} \mathrm{region} \mathrm{before} \mathrm{eruption} \mathrm{onset} \mathrm{and} \mathrm{dur-}$ ing the onset and explosive phase of the eruption. The imaged emission is from plasmas at temperatures around $1.5 \times 10^{6} \mathrm{~K}$ (Delaboudiniere et al. 1995). The bottom two panels are $\mathrm{SOHO/LASCO/C2} \mathrm{running-difference} \mathrm{images} \mathrm{before} \mathrm{and} \mathrm{after} \mathrm{the} \mathrm{resulting} \mathrm{CME} \mathrm{emerged}$ from behind the occulting disk of the $\mathrm{C} 2$ coronagraph. A SOHO/MDI magnetogram is overlaid on the second EIT coronal image. This shows that the eruptive arcade was the central arcade of a quadrupolar magnetic region having roughly the layout sketched in Fig. 5. Although the setup was right for breakout reconnection to have occurred at the top of the central arcade as it erupted, if it did occur, its heating was too weak to produce detectable side-arcade brightening in the EIT coronal images.

In the first EIT coronal image in Fig. 6, the central arcade has not yet started to erupt. A filament is suspended in the core of this arcade, and the arcade is seen as a coronal cavity enveloped in faint outer loops. The filament and the rest of the central arcade began slowly rising together around 8:00 UT, and by the time of the second EIT coronal image, about 45 minutes later, the height of the filament had increased by only 20\% (Sterling and Moore 2004). Then the eruption grew increasingly faster, the filament erupting to a few times its initial height by the time of the third frame, 24 minutes later. In this image, the flare ribbons heated by the tether-cutting reconnection below the erupting filament-carrying core-field flux rope are turning on near the tip of the black arrow and are still faint and small. Over the next 12 minutes to the last EIT coronal image in Fig. 6, the arcade and its filament exploded much farther out as the two flare ribbons grew brighter and much longer. Thus, while this eruption of the central arcade of a quadrupolar magnetic region shows no signatures of breakout reconnection in these images, it does show evidence that the current sheet that formed below the erupting filament for the tether-cutting reconnection was at first very small on the scale of the erupting arcade and then grew much larger as the arcade blew out, in the same manner as in our two example isolated-arcade eruptions.

Our next example embedded-arcade flare/CME eruption is similar to the eruption in Fig. 6 in that the eruptive arcade was the central arcade of a quadrupolar magnetic region. But this time the magnetic field was much stronger and the eruption started with obvious signatures of breakout reconnection. The quadrupolar magnetic region in Fig. 6 was the mostly spotless remains of two or more decayed sunspot active regions. The strongest field was in the eastern side arcade, which had the only remaining sunspot. Averaged over areas the size of a supergranule $(30,000 \mathrm{~km}$ across), the photospheric flux in the central arcade and in the western side arcade was nowhere stronger than $\sim 100 \mathrm{G}$, much less than 

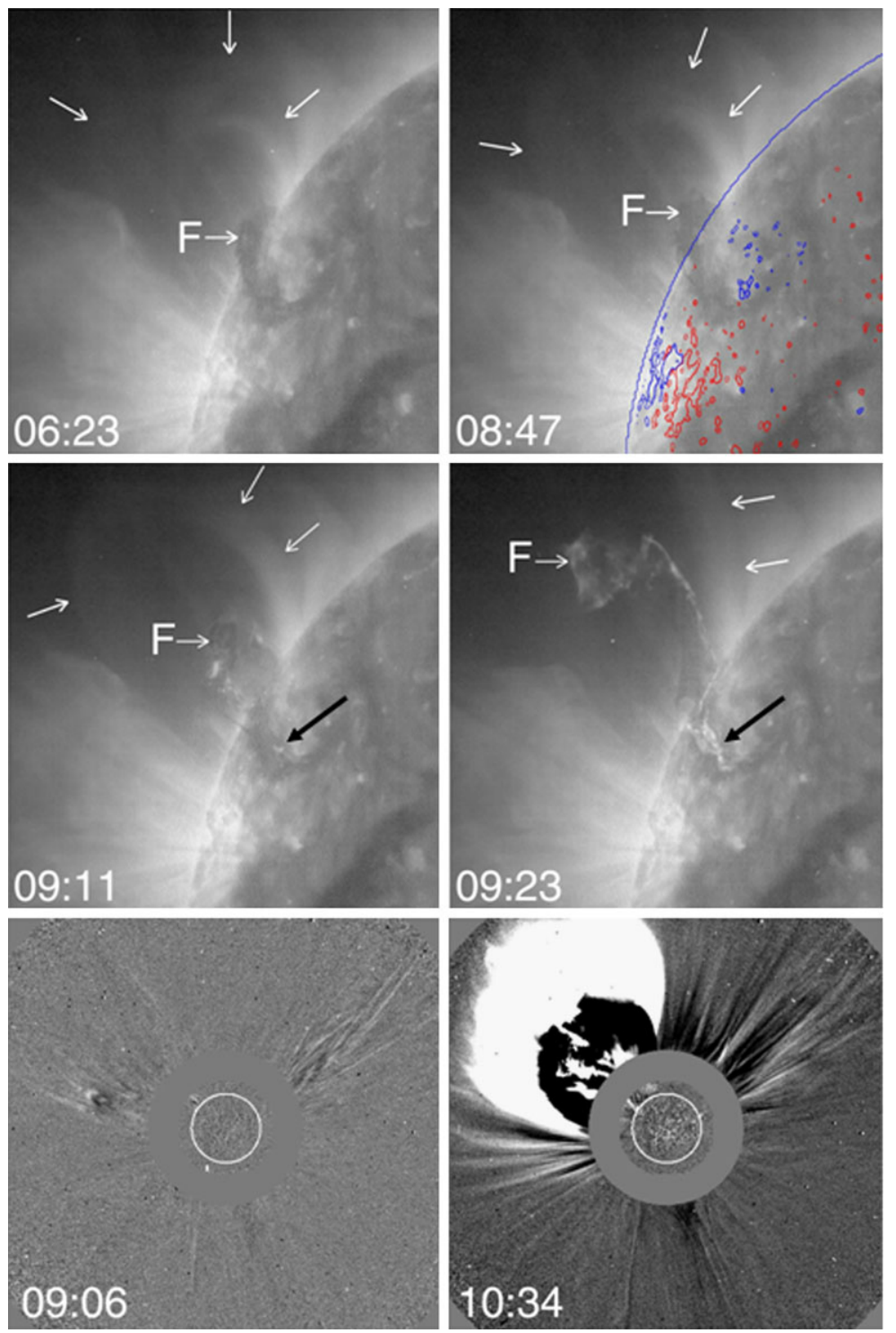

Fig. 6 Onset and explosive growth of an embedded-arcade ejective eruption observed by $\mathrm{SOHO}$ on 2002 January 4 (from Sterling and Moore 2004). See text. The unlabeled white arrows point to the outer edge of the eruptive arcade. The white arrow labeled $F$ points to the filament in the core of the eruptive arcade. In the overlaid MDI magnetogram in the second frame, positive-polarity flux is red and negative-polarity flux is blue. The black arrow in the third and fourth frames points to the growing flare ribbons along the neutral line of the eruptive arcade's magnetic flux. The universal time is in the lower left of each frame

the $\sim 1000 \mathrm{G}$ required for a full-fledged sunspot. In contrast, the quadrupolar magnetic region of our next example eruption was a still-growing large sunspot active region in which each of the four polarity domains had one or more sunspots, three of them having one or more major sunspots. So the field in the central arcade of this quadrupolar active region 


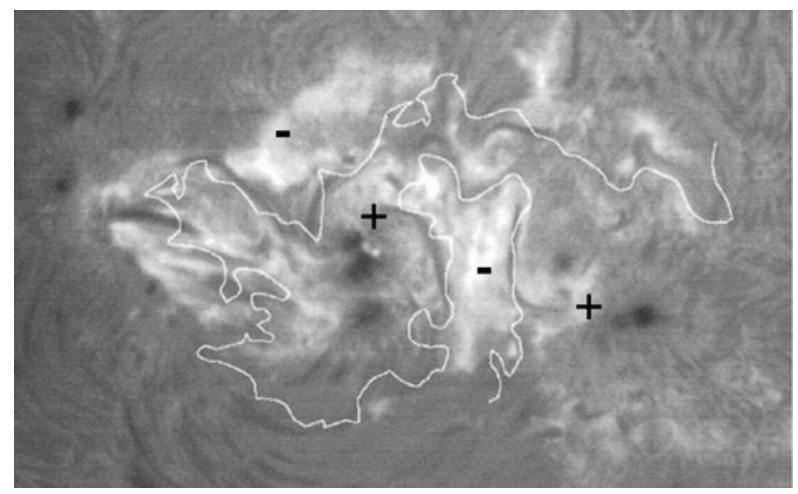

Fig. 7 Pre-eruption $\mathrm{H} \alpha$ image and polarity arrangement of the quadrupolar active region AR 10030 on 2002 July 15 (from Gary and Moore 2004). The white line is the polarity inversion line from an MDI magnetogram. The plus and minus signs mark the polarities of the four flux domains. The dark filament that runs along the neutral line between the inner pair of flux domains is about 40,000 km long, sits in the core of the central embedded arcade of the active region, and shows that the core field is greatly sheared and hence has much pent-up free energy. About 14 hours later this filament, carried in the core field, erupted and blew out with the rest of the arcade field that was unleashed by breakout reconnection early in the production of a major flare and $\mathrm{CME}$

was several times stronger than in the central arcade in Fig. 6. The quadrupolar arrangement of magnetic flux in this large strong-field active region on the day of the eruption is shown in Fig. 7, which is an $\mathrm{H} \alpha$ image of the pre-eruption active region on which is superposed the main neutral line extracted from an MDI magnetogram. The $\mathrm{H} \alpha$ image shows most of the larger sunspots, and the polarity of each of the four flux domains is shown by a plus sign or a minus sign. Thus, Fig. 7 shows that this active region (AR10030) was obviously quadrupolar in the manner of the cartoons in Fig. 5, and that the east-to-west (leftto-right) sequence of alternating polarity was the same as in the quadrupolar active region in Fig. 6. Because the active region was at north $19^{\circ}$ and west $1^{\circ}$ on the day of the eruption, it was viewed from above and slightly from its south side. The $\mathrm{H} \alpha$ image in Fig. 7 shows a dark filament sitting in the sheared-field core of the central arcade. This filament erupted as breakout-reconnection flare ribbons grew explosively, indicating that this eruption was initially unleashed by breakout reconnection as depicted in Fig. 5 (Gary and Moore 2004; Li et al. 2005).

In the same way as the $\mathrm{H} \alpha$ filament in Fig. 3, the $\mathrm{H} \alpha$ filament in the middle of the active region in Fig. 7 was a visible tracer of some lower strands of the central arcade's flux-rope core field before and during the arcade's blowout eruption. This eruption produced a major flare (GOES X3) and a large fast CME $(1100 \mathrm{~km} / \mathrm{s})$. The filament started erupting between 20:01 UT and 20:02 UT and was accelerated to an upward velocity of about $700 \mathrm{~km} / \mathrm{s}$ over the next three minutes as it was ejected up and out of the active region ( $\mathrm{Li}$ et al. 2005). The time profiles of the flare's soft X-ray flux and microwave flux are shown in Fig. 8. The first and strongest impulsive spike in the microwave burst peaks at 20:04 UT, in step with the steepest rise in the soft X-ray emission, as is typical. Both the microwave spike and the steep rise in the soft X-ray profile start about two minutes earlier, at about 20:02 UT, which was when the filament was starting to erupt. This timing is just that expected for the electron acceleration and plasma heating that produced the microwave spike and steep rise in soft X-ray emission to have been produced by a burst of reconnection that both unleashed the eruption and was driven by it. Figure 8 also shows that the X-ray flux started rising slowly 


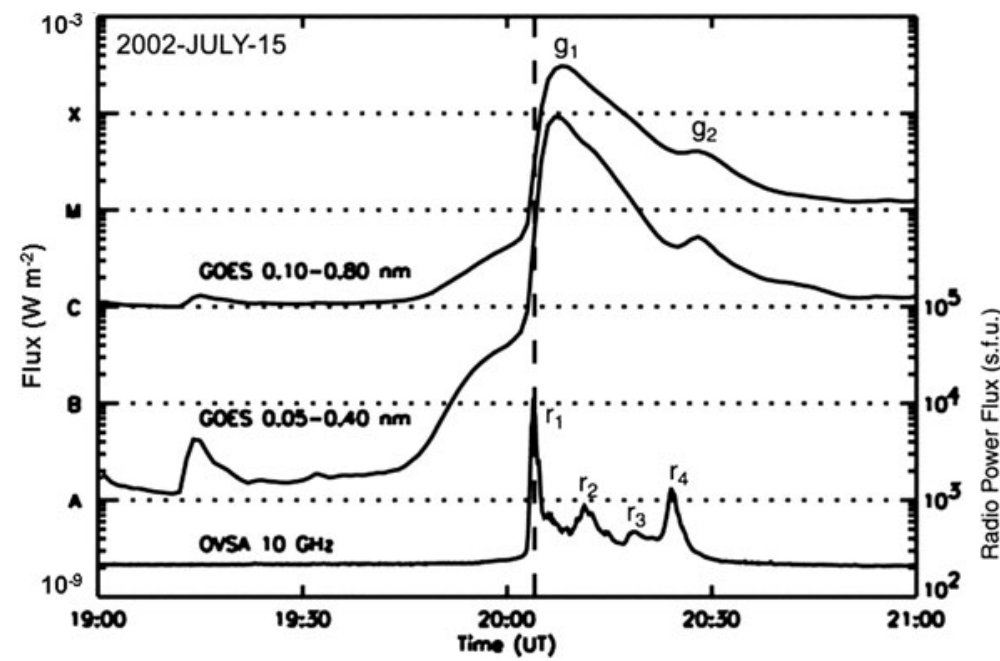

Fig. 8 Temporal development of the soft X-ray and microwave emission produced by the 2002 July 15 flare/ CME eruption of the quadrupolar active region in Fig. 7 (from Gary and Moore 2004). The upper two curves are the 1-8 $\AA$ and $0.5-4 \AA$ flux observed by GOES. The bottom curve is the $10 \mathrm{GHz}$ microwave flux observed by the Owens Valley Solar Array. The vertical dashed line marks 20:04 UT, the time of the peak of the tallest spike in the microwave flux and the time of the steepest rise in the soft X-ray flux
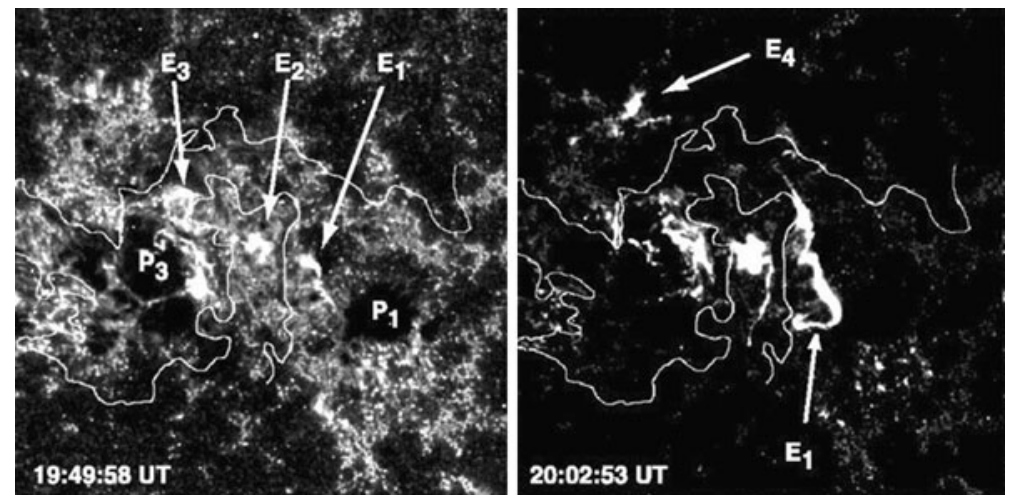

Fig. 9 Breakout-reconnection flare ribbons in TRACE $1600 \AA$ images before (left) and early in (right) the explosive fast phase of the 2002 July 15 flare/CME eruption of the central arcade of the quadrupolar active region in Fig. 7 (from Gary and Moore 2004). The overlaid white contour is the main polarity inversion line in the active region's magnetic flux, as in Fig. 7. The four flare ribbons, pointed out by the arrows and labeled $E_{1}, E_{2}, E_{3}, E_{4}$ from west to east, are the signature of breakout reconnection at the top of the central arcade. Each ribbon is discernible in the first image, and each is bigger and brighter in the second image

about 20 minutes earlier, gradually increasing several fold before the steep rise started. This is an indication that the burst of reconnection was preceded by weaker gradual reconnection.

The above inferences from Fig. 8 about reconnection before and during the fast rise of the flare's soft X-ray flux are supported by the breakout-reconnection flare ribbons shown in Fig. 9. Each of the two images in Fig. 9 is a TRACE $1600 \AA$ image on which the active region's neutral line has been overlaid. Four flare ribbons, one in each polarity domain, are present in both frames. Being well separated from each other, these four ribbons are appro- 
priate for being heated by breakout reconnection at the top of the central arcade, as depicted in Fig. 5. The first frame, at 19:49:58 UT, is during the slow rise of the soft X-ray emission prior to fast-eruption onset. At this time, the average length of the four ribbons is much shorter than the $\mathrm{H} \alpha$ filament in Fig. 7, indicating that during this early quasi-static gradual turn-on phase of slow breakout reconnection, the breakout-reconnection current sheet was small relative to the eruptive central arcade. The second frame of Fig. 9, at 20:02:53 UT, is about a minute after fast eruption started. Each of the four ribbons is now much brighter and the average length of the four ribbons is now comparable to the length of the pre-eruption filament in Fig. 7. Most strikingly, the western ribbon has grown three to four times longer than it was in the first frame. This ribbon continued its explosive growth, doubling in length over the next minute as the eruption continued to accelerate (see Fig. 7 of Li et al. 2005). This explosive growth of the breakout-reconnection flare ribbons in step with the explosion of the central arcade is evidence that the breakout-reconnection current sheet was forced to become much larger by the outward explosion of the arcade. So, again we have evidence that reconnection starts at a quasi-static growing current sheet when the current sheet is still small compared to the eruptive arcade, and grows much larger only dynamically, forced by the eruption unleashed by the reconnection.

A pair of flare ribbons that started close to the central neutral line, appropriate for heating by tether-cutting reconnection of the legs of the erupting sheared core field, were produced by this flare/CME eruption, but these ribbons did not start until the $\mathrm{H} \alpha$ filament had erupted to a height comparable to the length of the active region ( $\mathrm{Li}$ et al. 2005). This pair of ribbons started below a second erupting flux rope, a helical one that the TRACE $1600 \AA$ movie, with $2 \mathrm{~s}$ cadence, shows to form and start erupting as this pair of ribbons turns on, at about 20:04:20 UT. In this movie, the helical flux rope explosively erupts upward, accelerating to $700 \mathrm{~km} / \mathrm{s}$ in about a minute, during which the pair of ribbons explosively grows in area from a small fraction to a much larger fraction of the erupting arcade (see Fig. 1 of Gary and Moore 2004). The birth and eruption of the helix and the coordinated birth and growth of the underling flare ribbons signify that (1) the helix was formed and unleashed by tethercutting reconnection that heated the ribbons, and (2) this reconnection current sheet started small and rapidly grew much larger during the explosive growth of the eruption of the helix.

\section{Reconnection in Standard X-ray Jets}

Coronal X-ray jets are a subclass of the solar eruptions that can occur when a small bipolar magnetic arcade, a miniature active region, emerges in the feet of a high-reaching unipolar field, such as the ambient field in a coronal hole or in one leg of a large-scale ( $\gtrsim 100,000 \mathrm{~km})$ coronal loop. In two different ways, both involving reconnection with the ambient field, magnetic energy can be explosively released to eject plasma up into the corona along the ambient field. If the eruption makes the ejected plasma hot enough to be seen in coronal $\mathrm{X}$-ray movies such as from Yohkoh/SXT or from the Hinode X-Ray Telescope (XRT), the eruption is observed as an X-ray jet (Shibata et al. 1992). If the ejected plasma is heated only to subcoronal temperatures, the ejection can't be seen in coronal X-ray images but can be seen in EUV images and/or in visible-wavelength chromospheric (e.g., H $\alpha$ ) images and is then called an EUV or chromospheric jet, surge, or macrospicule (Shibata 2001; Moore et al. 2010).

If the emerging arcade has enough free magnetic energy in the form of field shear and twist in its interior, then an X-ray jet can be produced by the arcade undergoing a miniature version of a CME eruption. An X-ray jet of this type, a blowout jet, amounts to a 
miniature embedded-arcade ejective eruption in which the eruptive arcade is embedded in high-reaching unipolar field. The eruption of the emerging sheared-core arcade appears to be unleashed partly by breakout reconnection at the current-sheet interface between the unipolar ambient field and the opposite-polarity leg of the impacted arcade, and partly by tether-cutting reconnection of the legs of the erupting arcade as in major flare/CME eruptions initiated by breakout reconnection (Patsourakos et al. 2008; Pariat et al. 2009; Rachmeler et al. 2010; Moore et al. 2010). In these X-ray jets, the hot "flare" loops made at the outside edge of the arcade by the breakout reconnection are typically much smaller than the erupting arcade, indicating that the breakout-reconnection current sheet is of similarly small extent (Moore et al. 2010). So, most of the released free energy that drives a blowout jet probably comes from the sheared and twisted interior of the erupting arcade as in major CME eruptions initiated by breakout reconnection, rather than from the pre-eruption field sandwiching the current sheet at which the breakout reconnection sets in as the eruption starts.

Many of the X-ray jets that occur in the Sun's polar coronal holes are blowout jets, but most, about two thirds, are of another type, the type that was first recognized and that until recently was generally thought to be the only type (Shibata et al. 1992; Shibata 2001; Moore et al. 2010). In contrast to blowout X-ray jets, in these most common X-ray jets, standard X-ray jets, the interior of the emerging arcade remains quasi-static and inert as the jet is produced.

The now well established reconnection scenario for standard X-ray jets, first deduced by Shibata et al. (1992) from the structure and development of X-ray jets observed in coronal $\mathrm{X}$-ray snapshots and movies from Yohkoh/SXT, is schematically depicted by the sequence of drawings in Fig. 10. Shown there are the rudiments of the pre-eruption field configuration (left drawing) and the elements of the jet during (middle drawing) and immediately after (right drawing) the burst of reconnection that produces the jet. In the first drawing, a bipolar arcade is emerging into ambient unipolar field of negative polarity. Because the

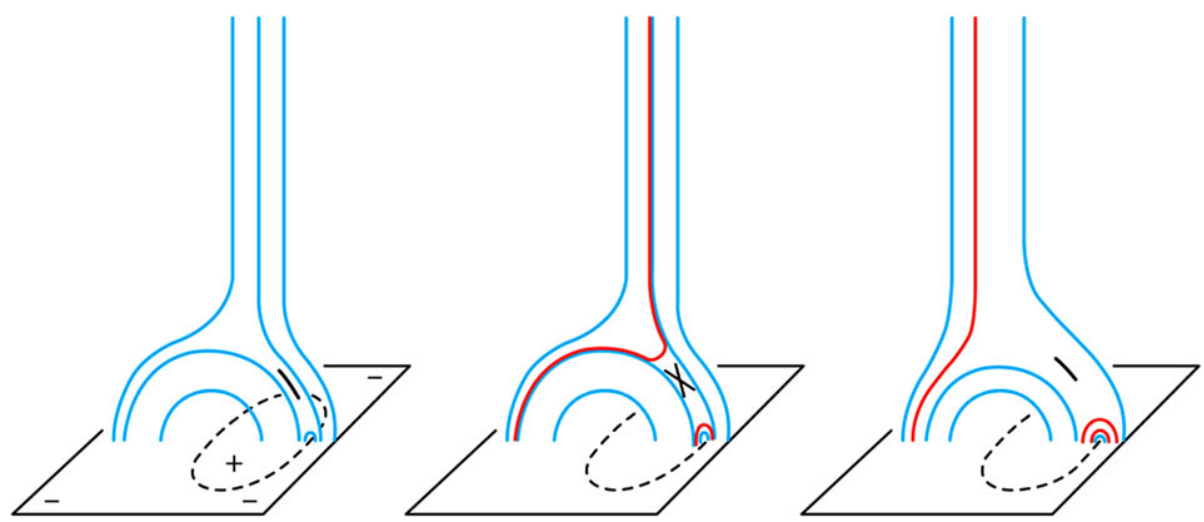

Fig. 10 Sequence of cartoons illustrating the field configuration, reconnection, and reconnection signatures in standard X-ray jets (from Moore et al. 2010). As in Fig. 5, only a few representative field lines (blue or red) are drawn. Left: The field configuration before the burst of reconnection that produces the jet. The dashed line is the polarity inversion line around the positive flux of the emerging arcade. The nearly unstable current sheet between the emerging closed field and the ambient open field is represented by the curved black line segment. Middle: The growing jet early in the burst of reconnection that is occurring in the disrupted current sheet and that is symbolized here by the slanted X. Red field lines are recently reconnected ones that consequently have hot plasma on them. Blue field lines either have yet to undergo reconnection or will not undergo reconnection. Right: The decaying jet immediately after the burst of reconnection has ended 
field in the positive-polarity leg of the arcade is opposite in direction to the ambient field impacted against it, a current sheet is built at their interface as the arcade emerges. For the amount of current in the sheet there is a corresponding amount of free energy in the compressed field sandwiching the current sheet. When it grows to be extensive enough and thin enough, the current sheet becomes unstable to reconnection and reconnection begins there, as depicted in the second cartoon in Fig. 10. The reconnection (1) releases upward open reconnected field lines that fling out the plasma on them to make the spire of the jet, and (2) releases downward closed reconnected field lines that build an arcade of hot "flare" loops over the neutral line at the positive-polarity end of the arcade. When the reconnection has released enough of the free energy in the compressed field sandwiching the current sheet, relaxing the field configuration nearly enough to the current-free potential-field configuration, the current sheet becomes stable again, reconnection stops, and the jet fades away.

In standard X-ray jets, and in blowout X-ray jets as well, the new hot arcade produced by the reconnection at the interface current sheet is typically much smaller than the emerging arcade. For this reason, this bright feature in X-ray images of an X-ray jet is commonly referred to as the jet's reconnection bright point (e.g., Savcheva et al. 2009).

In the standard model sketched in Fig. 10, and as appears to be the case in observed standard X-ray jets, all of the heating in the eruption results from the reconnection of the emerging arcade with the ambient field. That is, in standard X-ray jets there is no burst of magnetic activity and heating inside the emerging arcade as the jet is produced, whereas this is what is observed in blowout X-ray jets (Moore et al. 2010). The reconnection in a standard jet corresponds to the breakout reconnection in a blowout jet, but there is no blowout in a standard jet. That is, in a standard jet the interior of the emerging arcade remains stable and inert during the reconnection, whereas in a blowout jet the interior is ready to explode out and is unleashed to do so by this reconnection.

Figure 11 shows the onset, growth, and decay of a typical standard X-ray jet observed by Hinode/XRT in coronal X-ray images. The jet is inside the limb in the northern polar coronal hole. The magnetic-arch base of the jet is viewed about equally side-on and endon, its interior neutral line running from northeast to southwest at about $45^{\circ}$ to north-south. In the first frame, a previous jet has nearly decayed away and our jet has not yet started. At this time, the base arch is brightest in its core, heated to coronal temperatures by some internal process. In the second frame, 2 minutes later, the bright interior of the base arch is dimming, the spire of our jet is turning on at the northwest end of the base arch, and the reconnection bright point is turning on at the opposite end of the base arch, all of which matches the middle cartoon of Fig. 10. During the next 3 minutes (third and fourth frames), the interior of the base arch continues to fade while the bright point reaches its maximum size and brightness and the spire grows taller and brighter. That the interior of the base arch was fading and soon became invisible in coronal X-ray emission as the jet turned on and grew indicates that the core of the base arch did not actively participate in producing the jet. During the next 3 minutes after the fourth frame in Fig. 11, in the fifth and sixth frames, the bright point starts fading while the spire attains its greatest height and width. The spire then fades nearly to invisibility as the bright point continues to fade over the next and final 4 minutes covered in Fig. 11 (seventh and eighth frames). Thus, this jet fits the standard-jet scenario of Fig. 10 quite well.

In Fig. 11, the reconnection bright point starts small and at its largest is still much smaller than the jet's base arch. This signifies that the current sheet at which the reconnection occurred was small compared to the base arch when it became unstable to reconnection and was burned down by the reconnection in the production of the jet. 


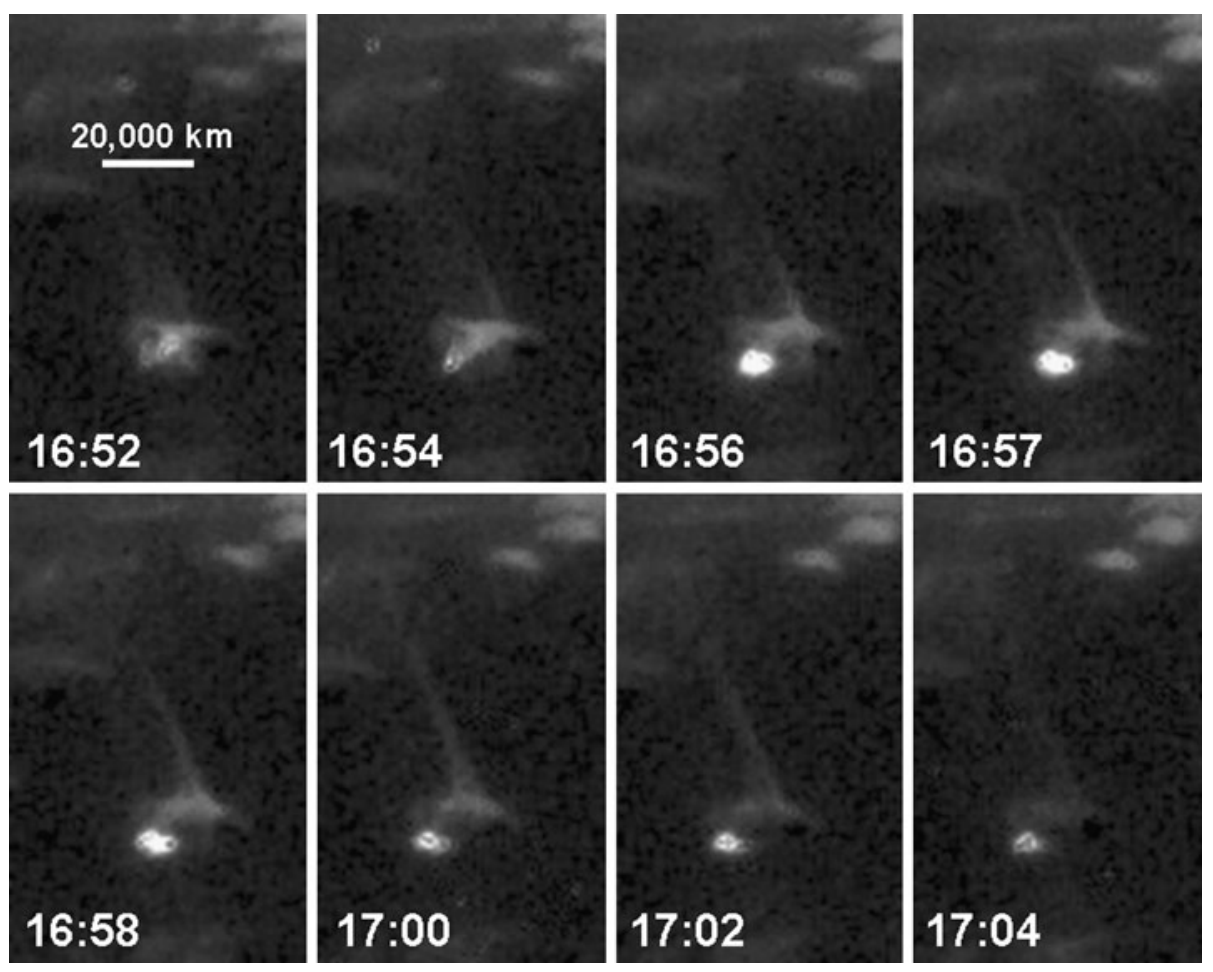

Fig. 11 Progression of a typical standard X-ray jet observed by Hinode/XRT (from Moore et al. 2010). These coronal X-ray images are centered on the jet and cover a sub field of view of selected frames from an XRT 1-minute-cadence movie of the northern polar coronal hole on 2008 September 22. The jet is near central meridian as can be seen from the slightness of the slant of the north limb, which is just inside the top edge of these images. The $20,000 \mathrm{~km}$ bar shows that the jet's base arch spans about 20,000 km. In the lower left of each frame is the universal time in hours and minutes

\section{Summary and Discussion}

In the solar eruptions that produce major flares and CMEs, the driving magnetic field is a sheared-core magnetic arcade that, before erupting, either stands alone or is embedded in the field of a multipolar larger active region. From the sweep of the arcade's pre-eruption interior field displayed in chromospheric and coronal images it is evident that the arcade has copious free energy stored in large-scale shear and twist in its interior field. In the middle of the arcade, the field direction near the neutral line is nearly parallel to the neutral line and the field direction changes only gradually toward perpendicular to the neutral line with distance from the neutral line. That is, there is no evidence of a large-scale pre-eruption current sheet inside the arcade. Most of the electric current corresponding to the arcade's free magnetic energy is apparently widely and smoothly distributed throughout the core field.

In the standard scenario for flare/CME eruptions depicted in Fig. 2, a small current sheet can form between the sheared legs of the sigmoid core field before the filament-carrying core-field flux rope begins to erupt. This current sheet is vertical and parallel to the neutral line and stands low in the core field above the neutral line and below the flux rope. The direction of the current in the sheet is horizontal and parallel to the neutral line. If this current sheet were not small, but instead had length and height comparable to the arcade, 
and there were as much sheet current as distributed current in the core field, then the field in the chromosphere near the neutral line would be rather steeply inclined to horizontal. That is, its vertical component would be comparable to its horizontal component. Instead, in $\mathrm{H} \alpha$ images having 1 arcsecond or better resolution (a few times better resolution than in Fig. 3 or Fig. 7), what is observed in the chromosphere in the core of a pre-eruption arcade is a so-called filament channel, a strip that is centered on the neutral line and has a width comparable to the height of the filament. The fibril structure of the channel shows that the field in the channel is nearly parallel to the neutral line and is nearly horizontal (e.g., see Martin 1998). This is evidence that if there is a current sheet low above the neutral line before eruption onset, it is of small extent compared to the arcade.

In flare/CME eruptions of either isolated arcades or embedded arcades, the onset and development of the pair of flare ribbons inside the erupting arcade indicates that a small current sheet forms in the sheared core field low in the middle of the arcade before, during, or soon after the onset of the eruption of the core-field flux rope. The two ribbons are typically faint, short, and close against the neutral line at first, and brighten and grow explosively with the eruption to span the length of the arcade as they spread apart. This progression indicates that the reconnection current sheet is initially small and dynamically grows much larger as an integral part of the accelerating eruption. Thus, both before and during eruption onset, there is good evidence that there is no arcade-sized current sheet in the pre-eruption arcade.

In embedded-arcade flare/CME eruptions and in blowout X-ray jets, the overall preeruption field configuration is such that there is oppositely directed field pressed against the top or side of the eruptive arcade. If the field around the interface were relaxed to its currentfree potential-field configuration, there would be a zero-field null point or null line on the interface. As the shear and twist builds up inside the arcade, the arcade puffs up, forcing a current sheet to form in place of the null. If the current sheet becomes extensive enough, it becomes unstable to reconnection and the resulting breakout reconnection unleashes the eruption of the arcade. In embedded-arcade flare/CME eruptions, the flare ribbons heated by the breakout reconnection typically start small in the slow-rise phase of the eruption and grow explosively with the eruption, as in our second example embedded-arcade eruption (Fig. 9). This progression of the breakout flare ribbons in that eruption is consistent with the breakout-reconnection current sheet being small at the start of the reconnection and then being forced to grow larger by the upward eruption of the arcade unleashed by the reconnection. Conceivably, the pre-eruption breakout current sheet was actually large, comparable in span to the eruptive arcade below it, and the reconnection started in a small patch of this large current sheet and quickly spread to the rest of the current sheet, producing the explosive growth of the breakout flare ribbons. If that were the case, most the free energy released during the explosive growth of the breakout ribbons would have been built up in the field sandwiching the current sheet before reconnection started. However, this possibility is unlikely in this eruption because the filament eruption showed that the core field was erupting fast and rapidly accelerating during the explosive growth of the breakout flare ribbons. That is, the entire arcade, including its deep core field, was exploding upward, ramming into the overarching field during the fast growth of the breakout ribbons. Therefore, the breakout current sheet was probably still small relative to the eruptive arcade when it became unstable to reconnection and started the eruption, and then grew rapidly, driven by the eruption. So, most likely, little of the magnetic energy released in the explosive phase of this eruption was in the pre-eruption field around the breakout current sheet.

In standard X-ray jets, in contrast to major flare/CME eruptions and blowout X-ray jets, the free magnetic energy released in the eruption evidently comes from the field sandwiching the current sheet between the pre-jet emerging arcade and the ambient open field, not from 
the interior of the emerging arcade. Reconnection at the interface current sheet generates the jet spire above it and a small flare arcade below it at the edge of the emerging arcade. The small size of this reconnection bright point indicates that the growing pre-jet current sheet becomes unstable to reconnection when it is still small relative to the driving arcade, and is then torn down by the burst of reconnection that produces the jet.

In conclusion, from the field configuration and the reconnection signatures in the onset and growth of the solar eruptions considered in this paper, we infer:

1. In major flare/CME eruptions and in X-ray jets, any current sheet present in the preeruption magnetic field is small relative to the driving magnetic arcade.

2. In major flare/CME eruptions and in blowout X-ray jets, a current sheet at which eruption-unleashing reconnection occurs can be driven to the size of the pre-eruption arcade by the unleashed eruption.

3. In major flare/CME eruptions and in blowout X-ray jets, the released free magnetic energy nearly all comes from the sheared and twisted magnetic field in the interior of the erupting arcade, and greatly exceeds the free magnetic energy corresponding to any preeruption current sheets.

4. In standard X-ray jets, the released free energy comes from the magnetic field sandwiching the gradually-built-up pre-eruption current sheet on the outside of the emerging arcade, not from the core of the arcade.

We conjecture that the above inferred characteristics of the reconnection current sheets in solar eruptions are basically consequences of the plasma $\beta$ (the ratio of plasma pressure to magnetic pressure) in the chromosphere and low corona in pre-eruption solar magnetic fields being much less than unity (Gary 2001). We reason this as follows. Consider the quadrupolar field configuration of Sweet in Fig. 1, and suppose that the field is initially relaxed to its current-free potential-field configuration. The potential field has an X-type null line between the two side arcades and above the middle arcade. The plasma $\beta$ is high $(\gg 1)$ near the null line because the magnetic pressure goes to zero at the null but the plasma pressure throughout is the ambient pressure of the hydrostatic atmosphere. Now, we start pushing the two side arcades together as Sweet imagined. At first the resulting current sheet in place of the null line will be very small and high- $\beta$ throughout. We suppose that as long as the plasma pressure in the current sheet greatly exceeds the pressure of the field pressing on the sides of the current sheet, the current sheet is stable against reconnection. If the two arcades have the field strength and plasma density of the coronal reaches of solar active regions, the plasma $\beta$ is very low $(\ll 1)$ everywhere but in a region that is centered on the null and is much smaller than either arcade. In this case, we expect that as the compression continues and the strength of the field pressing on the current sheet increases as the current sheet grows, the magnetic pressure on the current sheet exceeds the plasma pressure inside the current sheet when the current sheet is still small on the scale of the arcades. On this basis, we conjecture that in pre-eruption solar magnetic fields (1) when the plasma $\beta$ in the middle of a preeruption current sheet drops below some critical level of order 1, the current sheet becomes unstable to reconnection, basically because the plasma can no longer hold the two opposite fields apart, and (2) this critical $\beta$ is attained when the growing current sheet is still small compared to the driving arcade. Thus, the low- $\beta$ condition of the chromosphere and low corona is possibly the basic reason why the current sheet for a standard X-ray jet is still small compared to the emerging arcade when reconnection sets in to produce the jet, and why the breakout-reconnection current sheet is still small compared to the eruptive arcade when breakout reconnection starts in a flare/CME eruption or a blowout jet. Similarly, however a tether-cutting-reconnection current sheet is born in an eruptive sheared-core arcade, either 
by quasi-static evolution of the field before the eruption or dynamically early in the eruption, the low- $\beta$ condition is possibly the basic cause of reconnection setting in before the current sheet can become large.

Acknowledgements This work was funded by NASA's Science Mission Directorate through the Heliophysics Guest Investigators Program, the Solar and Heliospheric Physics Supporting Research and Technology Program, the Hinode Project, and the Living With a Star Targeted Research and Technology Program.

Open Access This article is distributed under the terms of the Creative Commons Attribution Noncommercial License which permits any noncommercial use, distribution, and reproduction in any medium, provided the original author(s) and source are credited.

\section{References}

S.K. Antiochos, Astrophys. J. 502, L181 (1998)

S.K. Antiochos, C.R. DeVore, J.A. Klimchuk, Astrophys. J. 510, 485 (1999)

A. Bruzek, in Physics of Solar Flares (NASA SP-50), ed. by W.N. Ness (NASA, Washington, 1964), p. 301

R.C. Canfield, H.S. Hundson, D.E. McKenzie, Geophys. Res. Lett. 26, 627 (1999)

H. Carmichael, in Physics of Solar Flares (NASA SP-50), ed. by W.N. Ness (NASA, Washington, 1964), p. 451

P.F. Chen, K. Shibata, Astrophys. J. 545, 524 (2000)

J.-P. Delaboudiniere, et al., Sol. Phys. 162, 291 (1995)

T.G. Forbes, J. Geophys. Res. 105, 23153 (2000)

G.A. Gary, Sol. Phys. 203, 71 (2001)

G.A. Gary, R.L. Moore, Astrophys. J. 611, 545 (2004)

J. Heyvaerts, E.R. Priest, D.M. Rust, Astrophys. J. 216, 123 (1977)

P.A. Isenberg, T.G. Forbes, P. Demoulin, Astrophys. J. 417, 368 (1993)

J.A. Klimchuck, in Space Weather, ed. by P. Song, H.J. Singer, G.L. Siscoe (AGU, Washington, 2001), p. 143

J. Li, D.L. Mickey, B.J. LaBonte, Astrophys. J. 620, 1092 (2005)

B.J. Lynch, S.K. Antiochos, C.R. DeVore, J.G. Luhmann, T.H. Zurbuchen, Astrophys. J. 683, 1192 (2008)

S.F. Martin, Sol. Phys. 182, 107 (1998)

R.L. Moore, Astrophys. J. 324, 1132 (1988)

R.L. Moore, J.W. Cirtain, A.C. Sterling, D.A. Falconer, Astrophys. J. 720, 757 (2010)

R. Moore, et al., in Solar Flares, ed. by P.A. Sturrock (Colorado Associated University Press, Boulder, 1980), p. 341

R.L. Moore, B.J. LaBonte, in Solar and Interplanetary Dynamics, ed. by M. Dryer, E. Tandberg-Hanssen, IAU Symp., vol. 91 (Reidel, Dordrecht, 1980), p. 207

R.L. Moore, G. Roumeliotis, in Eruptive Solar Flares, ed. by Z. Svestka, B.V. Jackson, M.E. Machado (Springer, Berlin, 1992), p. 69

R.L. Moore, A.C. Sterling, in Solar Eruptions and Energetic Particles, ed. by N. Gopalswamy, R. Mewaldt, J. Torsti (AGU, Washington, 2006), p. 43

R.L. Moore, A.C. Sterling, H.S. Hudson, J.R. Lemen, Astrophys. J. 552, 833 (2001)

E. Pariat, S.K. Antiochos, C.R. DeVore, Astrophys. J. 691, 61 (2009)

S. Patsourakos, E. Pariat, A. Vourlidas, S.K. Antiochos, J.P. Wuelser, Astrophys. J. 680, L73 (2008)

L.A. Rachmeler, E. Pariat, C.E. DeForest, S. Antiochos, T. Torok, Astrophys. J. 715, 1556 (2010)

D.M. Rust et al., in Solar Flares, ed. by P.A. Sturrock (Colorado Associated University Press, Boulder, 1980), p. 273

D.M. Rust, A. Kumar, Astrophys. J. 464, L199 (1996)

A. Savcheva, J.W. Cirtain, E.E. DeLuca, L. Golub, Astrophys. J. 702, L32 (2009)

N.R. Sheeley, R.A. Howard, M.J. Koomen, D.J. Michels, Astrophys. J. 272, 349 (1983)

K. Shibata, in Encyclopedia of Astronomy and Astrophysics, ed. by P. Murdin (IOP Publishing, Bristol, 2001), p. 3258

K. Shibata, et al., Publ. Astron. Soc. Jpn. 44, L173 (1992)

A.C. Sterling, H.S. Hudson, B.J. Thompson, D.M. Zarro, Astrophys. J. 532, 628 (2000)

A.C. Sterling, R.L. Moore, Astrophys. J. 613, 1221 (2004)

P.A. Sturrock, Nature 211, 695 (1966)

P.A. Sturrock, in Structure and Development of Solar Active Regions, ed. by K.O. Kiepenheuer, IAU Symp., vol. 35 (Dordrecht, Reidel, 1968), p. 471 
P.A. Sturrock, Solar Flares (Colorado Associated University Press, Boulder, 1980)

Z. Svestka, Solar Flares (Springer, Berlin, 1976)

P.A. Sweet, in Electromagnetic Phenomena in Cosmical Physics, ed. by B. Lehnert (Cambridge University Press, Cambridge, 1958), p. 123

H. Zirin, Astrophysics of the Sun (Cambridge University Press, Cambridge, 1988) 